\title{
TEMPERATURE AND WEATHER CORRELATES OF MYASTHENIC FATIGUE
}

\author{
S. BORENSTEIN \\ JOHN E. DESMEDT \\ Brain Research Unit, University of Brussels, \\ Brussels, Belgium
}

The myasthenic neuromuscular fail-
ure tested by nerve stimulation and recording of muscle responses is aggravated by local warming and relieved by local cooling. Small alterations of temperature can elicit quite striking changes. False-negative diagnostic tests are sometimes related to a spontaneous lowering of the muscle temperature, and temperature should be checked routinely. Clinical correlates are consistent in showing that the affected muscles are influenced by even small local temperature changes, such as occur in daily living. This suggests a number of practical recommendations which can help these patients.

\section{Introduction}

PATIENTS with myasthenia gravis vary a great deal in the extent and severity of muscle weakness and fatigue. The amount of paresis of the affected muscles is typically aggravated by exercise (postactivation exhaustion ${ }^{1}$ ) and improved by rest or by anticholinesterase drugs, but it has not been hitherto appreciated that small changes in muscle temperature also have a profound effect. Besides its interest for neuromuscular pathophysiology this finding suggests a number of practical points for the diagnosis and treatment of myasthenia.

In clinical neurophysiology and electromyography muscle temperature is recognised as a significant factor which can jeopardise diagnostic conclusions when not properly controlled. For example, the spontaneous fibrillation after denervation may not be recorded in a cold muscle with slightly impaired blood circulation. On the other hand, mild myotonic discharges are potentiated by local muscle cooling. Tissue temperature has a striking influence on motor and sensory nerve-conduction velocity which drops 
by about $3 \mathrm{~m}$. per second for each fall of $1^{\circ} \mathrm{C} .{ }^{2.3}$ Intramuscular temperatures of $28-31^{\circ} \mathrm{C}$ are by no means exceptional in the distal limbs of patients examined in a room at $22^{\circ} \mathrm{C}$, and the nerve-conduction slowing related to such cooling ought to be differentiated from the genuine reductions recorded in polyneuropathies. ${ }^{\circ}$ The distal motor latency of the muscle electrical response to a nerve stimulus is also much increased by cooling which prolongs the neuromuscular refractory period for repetitive electrical stimulation of the motor nerve.' Microphysiological data in animals would not have predicted that myasthenic neuromuscular block is dramatically aggravated by local warming ${ }^{6}$ and that it can be relieved by local cooling. Pharmacological studies have shown that the neuromuscular block by $d$-tubocurarine improves with cooling while the block by depolarising drugs such as decamethonium or succinylcholine is potentiated by cold. ${ }^{7}$ Althouph the molecular mechanisms of the block produced by a competitive inhibitor such as $d$-tubocurarine are entirely different from those in myasthenia gravis, ${ }^{,-10}$ the safety factor for neuromuscular transmission is reduced in both cases and adversely affected by even a small rise in the local temperature."

\section{Patients and Methods}

We have investigated thirty patients between 10 and 86 years old with an unambiguous diagnosis of myasthenia gravis. A wide spectrum of clinical severity was displayed in this series, from the mild localised myasthenia of recent onset to the generalised long-standing form of the disease. The diagnosis had been established by clinical signs of variable motor paresis influenced by rest and exercise, positive nerve-stimulation tests in the affected muscles, and clear improvement with anticholinesterase therapy. Six patients had improved after surgical thymectomy. The patients were deprived of their anticholinesterase therapy for the longest possible time before the nerve-stimulation tests, which involved supramaximal electrical stimulation of motor nerves and the cathode-ray oscilloscope recording of belly-tendon electrical responses in the corresponding muscles, ${ }^{2.4}$ In a few of the patients the isometric myogram of the adductor pollicis muscle was recorded with a straingauge system with minimal compliance." The intramuscular temperature was monitored with a needle thermistor inserted $1 \mathrm{~cm}$. below the skin surface, as close as possible to the tested muscle. Local warming was achieved by an infra-red lamp. Local cooling was induced by applying crushed ice cubes in a plastic bag to the skin. In the myographic studies the hand and forearm were entirely immersed in liquid paraffin oil at $4-10^{\circ} \mathrm{C}$ 
in a large 'Perspex' tank containing the Brown-Shuster myograph table.

\section{Results}

\section{Nerve-stimulation Tests}

Fig. 1 illustrates a nerve-stimulation test on two hand muscles in a woman of 26 with a severe generalised myasthenia. She had been deprived of her pyridostigmine ("Mestinon") therapy for 15 hours. In the first test, before any warming, the intramuscular temperature happened to be as low as $31^{\circ} \mathrm{C}$, and the decrement of the successive electrical responses evoked by 3 per second nerve stimulation was barely above normal range (reduction of fifth response normally does not exceed $9 \%$ with respect to the first in the same train at 3 per second $\%$. The same muscles displayed a striking post-activation exhaustion when re-tested at the same temperature after a standard exercise involving uninterrupted 3 per second nerve

cold

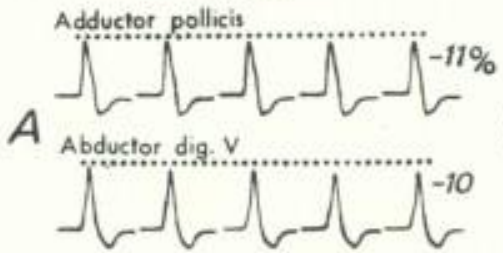

warm

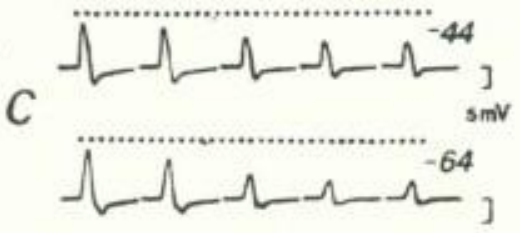

Exhaustion after $3 / \mathrm{sec}$ for $5 \mathrm{~min}$

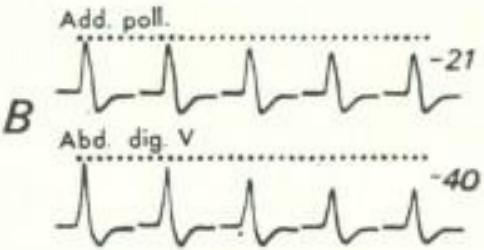

D
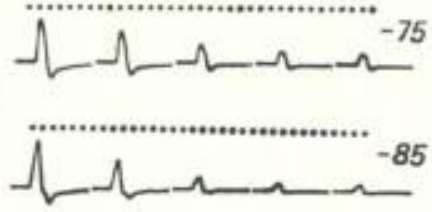

Fig. 1-Seimulation of the ulnar nerve at the elbow with supramaximal electrical pulses at 3 per second while recording the belly-tendon electrogram of the adduetor pollicis (upper trace) and of the abductor digiti quinti muscles in a myasthenic patient.

The decrement of the fifth response (relative to the first response of the same 3 per second train) is indicated in percent.

(A) Intramuscular temperature $31^{\circ} \mathrm{C}$. (B) Same test disclosing post-activation exhaustion after uninterrupted stimulation of the ulnar nerve at 3 per second for 5 minutes. (C) Same test after recovery and warming of the hand muscles to intramuscular temperature of $36^{\circ} \mathrm{C}$. (D) Same test at intramuscular temperature $36^{\circ} \mathrm{C}$ after the 5 -minute exercise. 

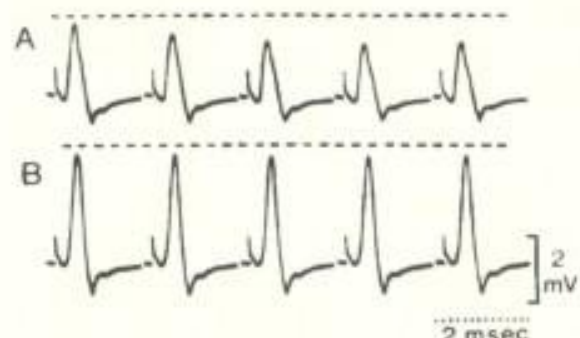

Fig. 2-Supramaximal stimulation at 3 per second of the facial nerve in front of the ear while recording the electrogram of the orbicularis oculi musele in a myasthenie patient. $30^{\circ} \mathrm{C}$.

(A) Intramuscular temperature $34 / 2^{\circ} \mathrm{C}$. (B) After cooling to

stimulation for 5 minutes (fig. 1B). The exhaustion is recorded as an increased decrement at 3 per second, the fifth responses being now reduced by -21 and $-40 \%$, respectively. About 40 minutes' rest was then allowed for recovery from post-activation exhaustion, and the muscles were warmed up to $36^{\circ} \mathrm{C}$ by an infra-red lamp. This resulted in a considerable aggravation of the neuromuscular block, the decrement being now 44 and $64 \%$ for the fifth responses in the 3 per second test. After the standard exercise at 3 per second for 5 minutes, the exhaustion resulted in decrements of 75 and $85 \%$.

Thus both the decrement in the rested muscles and the post-activation exhaustion were much increased when the muscles were warmed by only $5^{\circ} \mathrm{C}$. This example shows that false-negative diagnostic tests may arise in mildly affected cases when the muscle temperature has dropped spontaneously to unduly low values. The diagnostic yield of nerve-stimulation testing in neuromuscular diseases should be improved by working systematically on muscles kept warm at the normal range of $35-37^{\circ} \mathrm{C} .4$

The reverse test can readily be performed, and mild local cooling definitely improved the myasthenic neuromuscular failure. Fig. 2 shows electrical responses of the orbicularis oculi muscle during supramaximal electrical stimulation of the facial nerve at 3 per second. The decrement of the fifth response is $25 \%$ in the muscle at $34 \cdot 2^{\circ} \mathrm{C}$ (A). After cooling by local ice application to the skin overlying the orbicularis (B) the voltage of the muscle responses rose towards normal values and the decrement was as little as $4 \%$ (within the range of normal adults). When 

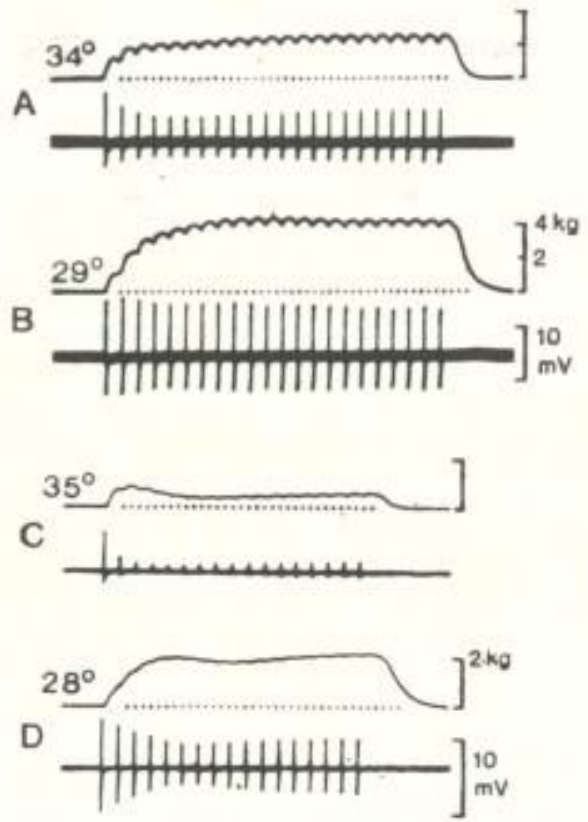

Fig. 3-Supramaximal stimulation of the ulnar nerve at 10 per second while recording simultaneously the isometric myogram (upper trace) and the belly-tendon electrogram of the adductor pollicis muscle.

(A, B) Male patient of 35 years with a moderately severe generalised myasthenia.

(C, D) Female patient of 20 years with a more severe generalised myasthenia.

Records at normal temperature and after local cooling are compared. The intramuscular temperatures are indicated for each test.

the myasthenic neuromuscular failure is improved by local cooling, the force of the muscle can be shown to be clearly increased, both in voluntary contractions and in mechanical recordings. Fig. 3 shows isometric myograms of the adductor pollicis muscle during supramaximal stimulation of the ulnar nerve at 10 per second, which is within the normal range of motor unit firing in moderate voluntary contractions. Two patients are illustrated, the first with a moderate generalised myasthenia (A, B) and the second with a more severe form of the disease (C, D). Local cooling in paraffin oil by about $5^{\circ} \mathrm{C}$ improves the electrical responses and increases the force output in both cases. 
The above findings indicate that even moderate local cooling reduces the neuromuscular block and improves the force output of a myasthenic muscle. Thermoregulation normally prevents the body temperature moving outside rather close limits, but local temperature changes can occur in body parts exposed to warmth or cold. The nerve-stimulationdata indicated that changes by only $2^{\circ} \mathrm{C}$ influence neuromuscular transmission to a significant extent. We therefore searched for possible clinical correlates in the thirty myasthenic patients.

\section{Clinical Correlates}

In seven patients with dysphagia, warm coffee, tea, or soup increased the difficulty in swallowing and sometimes increased the dysarthria also. Cold drinks and ice-cream made swallowing easier. This could be demonstrated by observing the patient when he drank water, first before and then after eating an ice-cream; in all cases swallowing improved. A few of these patients said they always allowed coffee or soup to cool down at meals. They insisted on drinking cold milk from the refrigerator when taking their first anticholinesterase tablet in the morning. By contrast the nineteen patients with no clinical evidence of dysphagia were not affected by hot or cold foods.

When asked about the preferred temperature of bath water, nineteen patients with myasthenia more or less generalised to limb and trunk muscles said that they were exhausted after a warm bath. They felt very weak and found it difficult to get out of the bath and to dress. One patient had been advised to take a Turkish bath and this seriously aggravated her pareses, leaving her helpless on a couch for about an hour. In view of these adverse effects the patients were using tepid water for baths or showers. Twelve of these patients also said they liked to wash their face with cold water because this improved their facial expression and reduced the dysmimia. Six other patients with a mild myasthenia localised to a few cranial muscles said they had no objection to a warm bath.

Exposure to bright sunlight had adverse effects in most myasthenic patients. In sixteen patients ptosis of the upper eyelid was rapidly elicited or increased. In seven myasthenics double vision or paresis of the orbicularis oculi appeared or increased in sunlight. As a result these patients all wore sunglasses in sunny weather. When assessing the adverse effect of bright 
sunlight it must be remembered that the orbicularis oculi muscles may become weaker, not only by local warming but also by exhaustion resulting from steady contraction to protect the eyes from the sun glare. On the other hand, the increased failure of the levator palpebræe and oculomotor muscles is likely to result primarily from local warming of orbital contents. Two patients had also experienced a severe generalised weakness during one session of sunbathing which they did not want to repeat. On the other hand, three patients with no clinically demonstrable involvement of the eye muscles were not affected by sunlight. Another patient with a quasi-permanent ptosis of the eyelids and severe oculomotor paralysis did not notice any worsening effect of sunlight; we suggest that her muscles were already exhausted and could not be made worse by local warming. Paresis of the lips and other facial muscles on exposure to the sun was occasionally described.

Seventeen patients with more or less generalised myasthenia did not feel so well during the warm summer weather as during the winter or spring months. They objected especially to hot stuffy weather. Some patients were weaker and in poor shape on summer holidays in Portugal, Spain, or Italy, while they improved on their way back when they stayed in the colder climate of the Alps. One patient experienced a myasthenic crisis with first appearance of diplopia and dyspncea while staying at the seaside in Rumania during a very hot summer. By contrast most patients could perform unusually well in bright cold weather, and several of them even had a good experience with winter sports. A young myasthenic girl was able to ski and play in the snow in the Alps, while she had difficulties climbing stairs at home. Exposure to cold wind is tolerated by some patients but not at all by others. Very cold weather, especially with humidity, is detrimental, one reason being, perhaps, that the muscle activity associated with thermogenesis contributes to post-activation exhaustion.

The climate of the Belgian seaside is similar to that of British sea resorts, and it proved beneficial for nine of our patients with generalised myasthenia. They claimed to be able to walk along the shore for, say, an hour, which would not have been possible at home. A few patients said they required less anticholinesterase therapy while at the seaside. One patient was remarkable in enjoying swimming in sea 
water at $18^{\circ} \mathrm{C}$. The patients, however, disliked the North Sea climate on the days with a cold heavy wind.

When asked about the preferred inside temperature at home, ten patients with generalised myasthenia said that they used central heating sparingly and set the room thermostat at $17-19^{\circ} \mathrm{C}$. Patients with mild localised myasthenia did not express any definite preference for reduced heating, and two of them actually liked a warm home at $22-23^{\circ} \mathrm{C}$, to which they had been accustomed since childhood before the onset of myasthenia. One professional cook complained of increased weakness when exposed to the heat of his oven.

\section{Discussion}

The clinical observations are consistent with the nerve-stimulation data, and it is surprising that temperature and weather correlates of myasthenic weakness have not been recognised previously. Patients may fail to notice temperature effects because their symptoms fluctuate with other variables such as rest and exercise, therapy, and the menstrual cycle. The fact that temperature changes affect only the muscles involved by myasthenia may have confused the picture since patients with a mild localised myasthenia may not notice temperature effects. We only identified these features progressively by detailed inquiries inspired by suggestions offered by our more attentive and critical patients, and we convinced ourselves by cross-examinations and nerve-stimulation studies. Given a choice of hot soup or ice-cream, a patient's response was unambiguous only if he had clinical signs of dysphagia, and grip strength was much improved by local application of crushed ice only if the patient had weak hand muscles. When properly tested, the improvement by local cooling about matches that after administration of prostigmine or pyridostigmine. These data emphasise the local effects on neuromuscular transmission, the improvement by cold involving presumably both an increased motor endplate effect of acetylcholine, and a reduced release of acetylcholine from the motor axon terminals, thus sparing the inadequate transmitter supplies. ${ }^{4.10}$ A more general influence of weather on the disease is only apparent for extremes of heat and cold (especially a heavy cold wind or hot stuffy weather) when the patient is stressed by 
thermoregulation demands.

A number of practical suggestions can be propored. For diagnosis it is advisable to test the mild or doubtful cases when the muscles are warm, this recommendation being exactly the opposite of the one for identifying myotonia. Local exposure of myasthenic muscles to cold may offer interesting clues-for example, improved swallowing after ice-cream or cold milk may help to distinguish a myasthenic from a neurogenic causation. These and other tests are harmless and may be fun to use in addition to usual nerve-stimulation procedures. Therapeutic advice for daily living should also be given-namely, advice on home temperature, avoidance of warm water in the bathroom, use of cold foods, choice of holiday resorts, wearing of sunglasses. Local warming in relation to physical treatment can be recommended in muscular dystrophy, ${ }^{\mathrm{n}}$ but would not help in myasthenia gravis.

This research has been supported by the Muscular Dystrophy Associations of America Inc, and by the Fonds de la Recherche Scientifique Médicale.

\section{REFERENCES}

1. Desmedt, J. E. Naturt, 1957, 179, 156.

2. Lambert, B. H. Electroenceph. clin. Nearophysial. 1962, suppl. 22. p. 9.

3. Kaeser, B. H. in Handbook of Clinical Neurology (edited by P. J. Vinken and $G$. W. Bruyn); vol. vn, p. 116. Amaterdam, 1970.

4. Deamedt, J. E. Acta neurel. belg. 1958, 58, 977.

5. Gilliatt, R. W. in New Developments in Blectromyography and Clinical Neurophysiology (edited by J. E. Desmedt); wol. II, D. 2. Basle, 1973.

6. Borenstein, S., Desmedt, J. E. ibid. vol. I (edited by J. E. Deamedt); p. 350 . Basle, 1973.

7. Cannard, T. H., Zaimis, E. J. Physiol., Lend. 1959, 148, 112.

8. Desmedt, J. E. Nature, 1958, 182, 1673.

9. Elmqvist, D., Lambert, E. H. Mayo Clin. Prac, 1968, 43, 689.

10. Desmedt, J. E. in New Developments in Electromyography and Clinical Neurophysiology (edited by J. E. Desmedt); vol, I, p. 305. Basle, 1973.

11. Demos, J. Am. F. phos. Med. 1971, 50, 271. 